\title{
Multiple myeloma and pulmonary aspergillosis: dental treatment prior to chemotherapy and management of post extraction complications
}

\author{
Amy Patrick, ${ }^{1}$ Khadeeja Saeed, ${ }^{2}$ Navdeep Kumar ${ }^{2}$
}

'Oral Surgery, Royal National ENT and Eastman Dental Hospital, University College London Hospitals NHS Foundation Trust, London, UK ${ }^{2}$ Special Care Dentistry, Royal National ENT and Eastman Dental Hospital, University College London Hospitals NHS Foundation Trust, London, UK

\section{Correspondence to \\ Dr Amy Patrick;}

amy.patrick@nhs.net

Accepted 1 April 2020

Check for updates

(c) BMJ Publishing Group Limited 2020. No commercial re-use. See rights and permissions. Published by BMJ.

To cite: Patrick A, Saeed K, Kumar N. BMJ Case

Rep 2020;13:e235038.

doi:10.1136/bcr-2020-

235038

\section{SUMMARY}

This case study discusses the dental management of a patient with a history of multiple myeloma and pulmonary aspergillosis, whom was referred to a hospital-based dental service for urgent dental review. The patient had received a dental assessment in primary care prior to commencement of chemotherapy and had four teeth extracted without complications. However, following the commencement of chemotherapy, he presented with a significant infection associated with two of his wisdom teeth resulting in extraction. Despite atraumatic extraction, the upper right wisdom tooth socket developed an oroantral fistula. A multidisciplinary team approach was required to enable effective patient management in this complex patient regarding myeloma, aspergillosis and the medications used including bisphosphonates and chemotherapy. It highlights the higher risk of oral complications that can arise in myelosuppressed patients and emphasises the need to identify potential sources of dental infection prior to the commencement of chemotherapy.

\section{BACKGROUND}

Multiple myeloma (MM) is a malignant haematological condition of the bone marrow where there is a proliferation and malignant transformation of plasma cells. ${ }^{1}$ These can cause osteolytic lesions in bone, with the potential for spread to adjacent tissues and organs. ${ }^{2}$ This condition represents $2 \%$ of all malignancies and $13 \%$ of haematological malignancies. Its incidence increases with age and can affect multiple body systems including bone, immune and neurological systems. ${ }^{3}$ Despite rapid advances in myeloma treatment which use stem cell transplants and immunomodulatory and cytotoxic properties of natural killer cells, at present, there is no effective cure for patients requiring long-term treatment. ${ }^{1}$

Aspergillosis is a rare fungal infection caused by Aspergillus, a species of mould that is found all over the world. ${ }^{4}$ It is a serious infection occurring more frequently in people with a compromised immune system and can be invasive, with associated pneumonia. ${ }^{5}$ The maxillary sinus is the most common sinus to be affected. Patients with pulmonary aspergillosis can present with fever and weight loss, and symptoms can vary and include chest pain, productive chesty cough, malaise and wheezing. ${ }^{5}$ These symptoms can make patients feel even more fatigued as well as heightening their risk of other infections. Close monitoring of patients is required to ensure early management of systemic infections to prevent detrimental decline in health. Furthermore, there is a risk of relapse if there is concurrent myelosuppression. $^{6}$

A detailed preoperative risk assessment and coordinated team approach are required in relation to essential dental extractions for a patient who presents with a history of MM and aspergillosis. It is vital that any dental treatment required is communicated to the haematological team to ensure appropriate patient management.

Myelosuppressed patients have an increased risk of developing oral infections, which can become life threatening. In view of this, detailed dental assessment prior to the commencement of intensive chemotherapy is essential to ensure potential causes of oral infection can be eliminated. This case study describes the dental management of a patient with a history of MM and aspergillosis. It also demonstrates the complications that can occur if dental extractions are undertaken after the commencement of intensive chemotherapy. ${ }^{7}$

\section{CASE PRESENTATION}

A man in his 50s was referred to a hospital dental department by his general dental practitioner (GDP) for pain from his upper right and lower right third molars (wisdom teeth). The patient's medical history included MM diagnosed in 2017, for which he had received 10 previous sessions of $30 \mathrm{~Gy}$ total dose radiotherapy to his right clavicle. Prior to chemotherapy, the patient had four uneventful dental extractions with his GDP. He then commenced intravenous bortezomib, a proteasome inhibitor used for the treatment of MM, with additional medications summarised in box 1 . The patient also received zoledronic acid infusions every month for 5 months, which were discontinued on presentation with a dental infection. Other relevant medical factors included the diagnosis of chronic cavitary pulmonary aspergillosis in 2015 (managed with long-term ambisome), chronic sinusitis and chronic bronchiectasis.

A thorough dental examination confirmed that there was evidence of chronic periodontal disease with generalised supra-gingival and subgingival calculus deposits present. The lower right wisdom tooth (LR8) was grade 3 mobile with a buccal 


\section{Box 1 Medications}

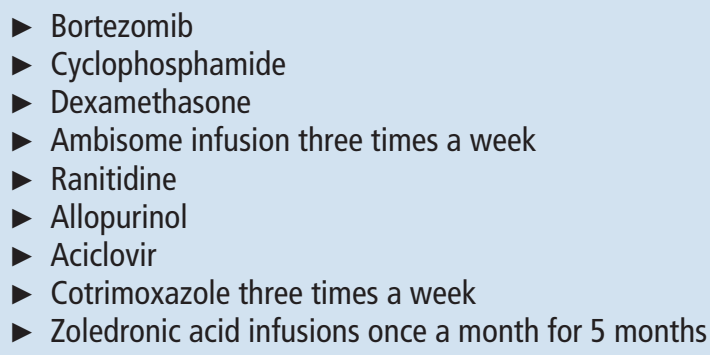

periodontal abscess and the upper right wisdom tooth (UR8) was grade 1 mobile with an associated $9 \mathrm{~mm}$ vertical pocket mesially.

An orthopantomogram (OPG) was undertaken to confirm the diagnoses (figure 1). It demonstrated that although there was a generalised horizontal bone loss, this was much more advanced in relation to the LR8 and UR8, with the LR8 associated with an extensive periapical area. The changes were indicative of periodontal disease exacerbated by risk factors (myelosuppression), rather than osteolytic changes linked to $\mathrm{MM}$ or pre-existing medication-related osteonecrosis of the jaws (MRONJ). The OPG did not demonstrate an opacification of the right maxillary sinus and the roots of the UR8 did not appear to be in close approximation to the sinus floor. A definitive diagnosis of chronic apical periodontitis with perio-endo lesions of the LR8 and UR8 was established.

Assessment of the patients' blood parameters and profiles, current MM treatments and discussion with haematological medical team allowed careful multidisciplinary treatment planning on the same day. An urgent blood test demonstrated a normal full blood count, as the patient was in the recovery week following 3 weeks of chemotherapy. The haematology team confirmed that dental extractions could be undertaken at

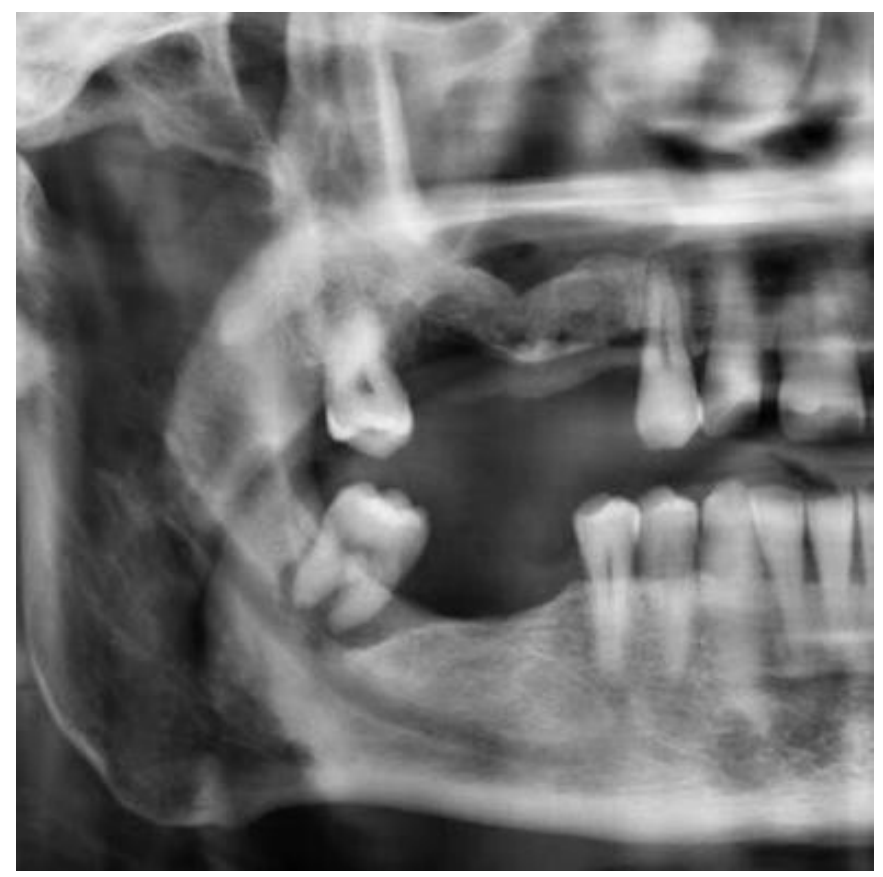

Figure 1 Sectional OPG shows LR8 and UR8 with extensive mesial and periapical radiolucencies correlating with the clinical findings of associated infection and periodontal disease. OPG, orthopantomogram; LR8, lower right wisdom tooth; UR8, upper right wisdom tooth.

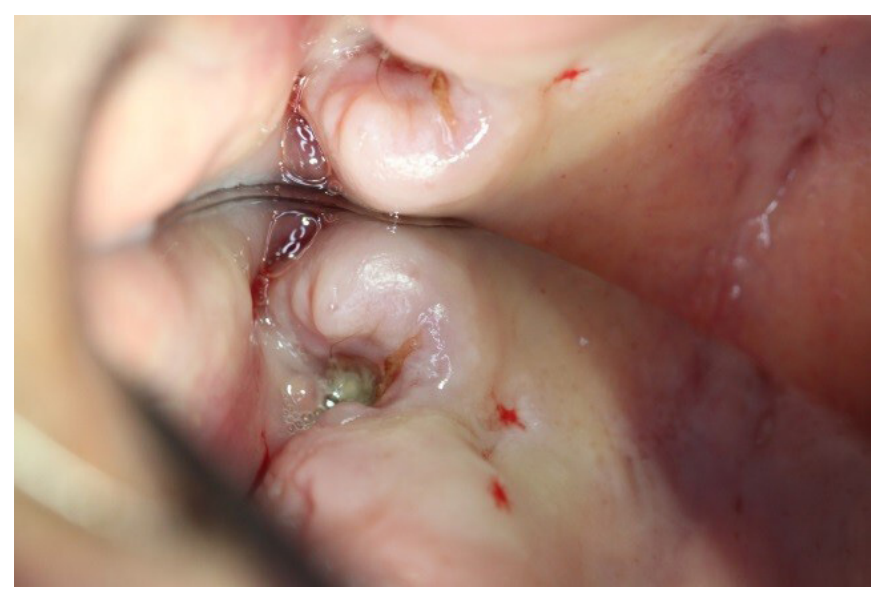

Figure 2 UR8 socket region: non-healing socket (reflection in a photographic mirror). UR8, upper right wisdom tooth.

this time and that chemotherapy would be delayed by 1 week to allow for healing. The LR8 and UR8 were extracted successfully with local anaesthesia without bone or soft tissue removal.

At the review of 1 week postextraction, there was no residual pain, swelling or discharge from the sockets. The patient proceeded with chemotherapy 1 week after the dental extractions. Due to the history of zoledronic acid infusions and the risk of MRONJ, a review appointment was arranged at 8 weeks.

At the second review appointment, the LR8 socket had healed fully. Although the patient was apyrexial, he was feeling fatigued and lethargic. The patient also reported increasing discomfort in the region of the upper right maxillary sinus, with the overlying facial tissues tender on palpation. There was an associated yellow discharge from the nose and the patient reported that he had a yellow/red discharge from the mouth when the soft tissues in the upper right quadrant were 'sucked'. Clinically, there was evidence of a non-healing socket in the UR8 region associated with a $10 \mathrm{~mm}$ vertical probing depth through the socket and potential communication with the maxillary sinus (figure 2). There was no evidence of sequestrum or spread across fascial planes.

\section{INVESTIGATIONS}

A culture and sensitivity swab was taken of the discharge fluid from the UR8 socket, which confirmed moderate Gram-positive cocci and Gram-negative rods with no fungal growth.

Cone beam CT images were taken to assess sinuses and showed unilateral and almost complete opacification of the right maxillary sinus with some bony destruction at the site of the UR8 (figure 3). A previous CT image of the maxillary sinuses was undertaken in 2016 and reviewed. The time of opacification of the right maxillary sinus was also noted but thought to be due to chronic sinusitis rather than linked to aspergillosis.

\section{DIFFERENTIAL DIAGNOSIS}

It was thought that the delayed healing of the UR8 could be related to concurrent myelosuppression or MRONJ. A biopsy of the affected bony tissues may be able to differentiate these diagnoses.

The clinical investigations also showed a $10 \mathrm{~mm}$ pocket, however, preoperatively, there was a $9 \mathrm{~mm}$ pocket that may simply indicate an established bone loss and delayed or 


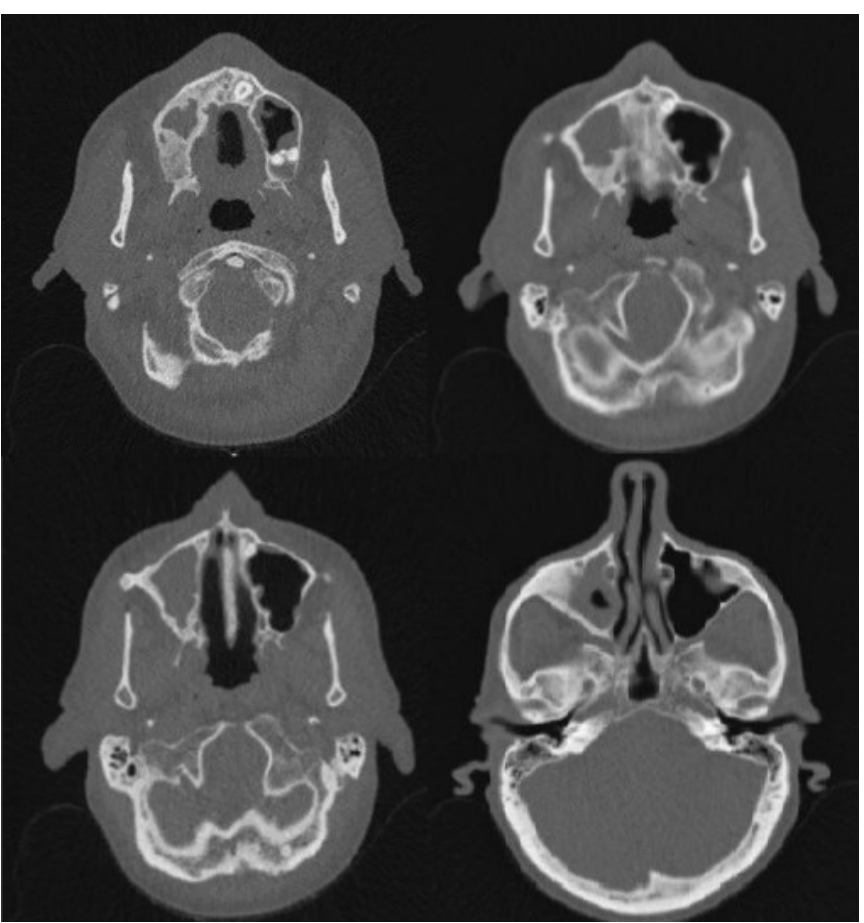

Figure 3 CT images taken to assess sinuses. Images show almost complete opacification of the right maxillary sinus with some bony destruction at the site of the upper right eighth tooth cavity.

incomplete soft tissue healing, or could indicate the presence of an oroantral fistula (OAF) with associated maxillary sinusitis.

Reactivation of aspergillosis involving the right maxillary sinus causing breakdown of the antral floor, and thus an OAF was also possible.

\section{TREATMENT}

The patient was managed across special care dentistry and oral surgery for closure of the OAF 1 week after diagnosis and regular postoperative review, with close communication with his haematology team. The surgical approach preferred to close the OAF was a single buccal advancement flap as it was decided that this would allow the defect to be repaired, with the option of furthermore extensive repair if required at a later stage. This decision considered the risk of buccal fat pad atrophy and potentially aggressive infection that may result in poor healing and breakdown if a more extensive approach was used at the outset.

The minimally invasive procedure was successful with the buccal flap raised, advanced and a positive seal obtained (figure 4). A 7-day course of $500 \mathrm{mg}$ clarithromycin two times per day was prescribed, based on the advice from microbiology. At the review of 2 weeks after closure, the patient reported that he had continued to 'suck' the pus from the sinus through the OAF, resulting in a partial breakdown of the wound. He was advised against this and reviewed regularly at 2 -week intervals. The OAF gradually reduced in size as it closed by secondary intention with the patient reporting feeling well in himself, with little fatigue or previous symptoms of aches or swelling in the area. At 8 weeks post closure, clinical examination demonstrated the OAF was still present; it was felt at this point surgical closure should be attempted again this time using the buccal fat pad. During the procedure, the fat pad was minimal and tethered showing signs of atrophy. Ordinarily it could be dissected towards the temporalis, however, for this case, specifically this intervention would have significantly increased the risk of infection, and so

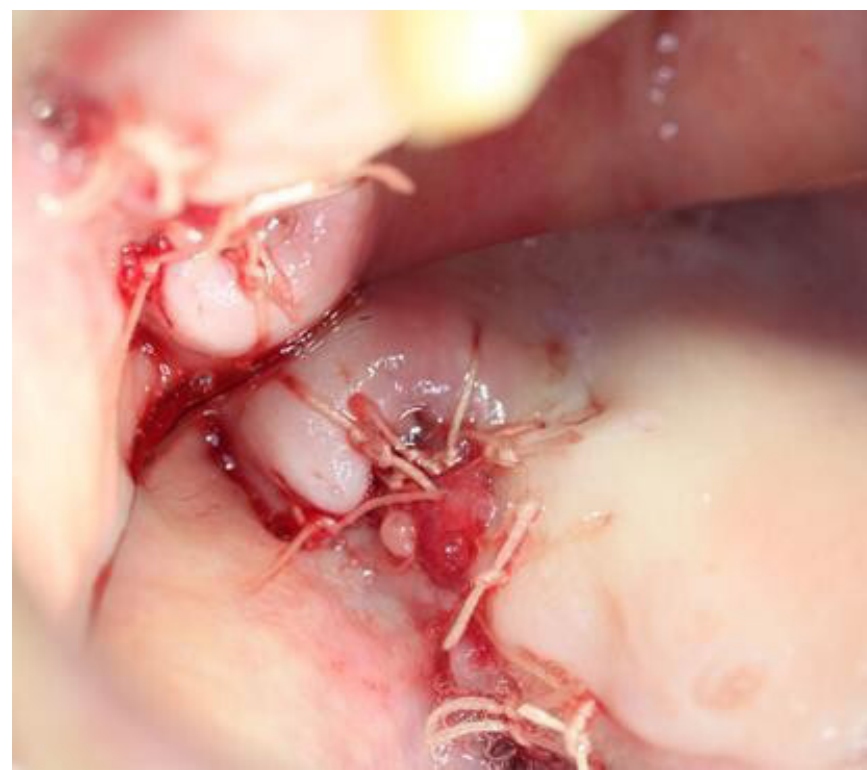

Figure 4 UR8 region: postdebridement and closure of OAF (reflection in a photographic mirror). OAF, oroantral fistula; UR8, upper right wisdom tooth.

the flap was closed similarly to the original procedure. In order to achieve optimal closure and reduce infection risk, the exposed bone was trimmed and smoothed-samples were sent to histopathology, which confirmed a mixture of live and necrotic bone, thus a diagnosis of MRONJ was confirmed.

\section{OUTCOME AND FOLLOW-UP}

The patient was regularly reviewed and the OAF continued to heal. The CT sinuses on 1-year review show a thickened lining with no sequestrum or OAF (figure 5).

\section{DISCUSSION}

This case highlights the complex diagnostic and dental decisionmaking required in a patient who presents with oral infection after the commencement of chemotherapy. Several factors had to be considered including the underlying haemato-oncological disease, chemotherapy, history of bisphosphonates and aspergillosis. Furthermore, it demonstrates the complications that can occur postdental extractions, where there are multiple potential causes and contributory factors. The challenges included delayed healing post dental extraction, an OAF, maxillary sinus opacification with related symptoms of discomfort and discharge.

MM can affect many body systems and leave patients immunocompromised and susceptible to infection. This can ultimately affect or delay their cancer treatment. ${ }^{8}$ Furthermore, MM can cause osteolytic lesions in bone and can be seen in the spine, lumbar region and jaw. ${ }^{3}$ Any radiolucency found in bone in special tests must be investigated to ensure there is no malignant cause. $^{4}$

This patient had multiple comorbidities including pulmonary aspergillosis, which resulted in chronic symptoms of fatigue and chest infections. He required urgent dental extractions due to dental pain and infection, which were carried out successfully but were followed by a delayed presentation of OAF in the upper extraction site. The patient had persistent discharge from the area after habitual 'sucking' post dental extractions. The importance of patients following postoperative instructions is vital and can influence healing. 


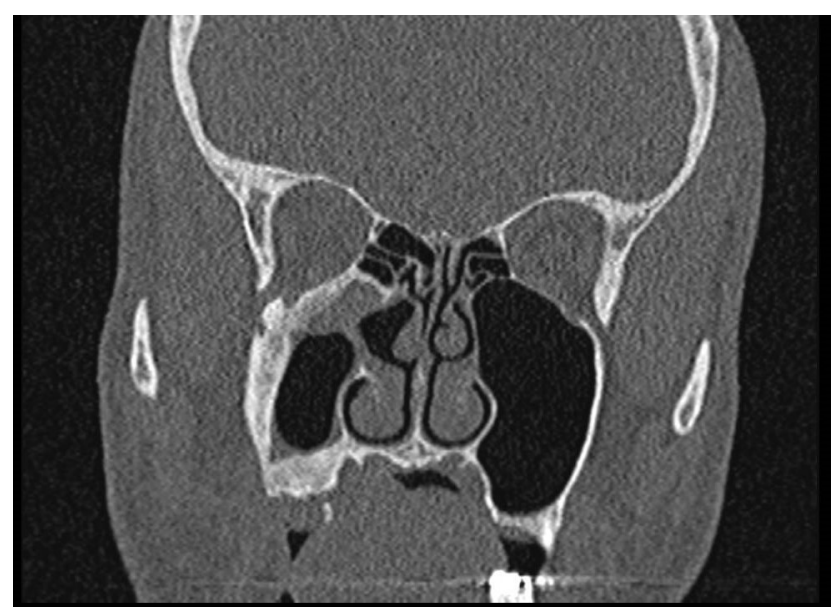

Figure $5 \mathrm{CT}$ images showing clear sinuses, no OAF and no sequestrum. OAF, oroantral fistula.

The complication of osteonecrosis of the jaw secondary to medications include drugs such as bisphosphonates, antiangiogenics and drugs for cancer therapy, and is well documented in the literature. ${ }^{89}$ With increased awareness among the dental team, more patients on these medications are being informed of the risks of MRONJ prior to any invasive dental procedure and are reviewed postoperatively to allow early intervention for any associated complications. Furthermore, a study by McGowan et al found the incidence of MRONJ in patients on multiple medications with systemic comorbidities was higher. ${ }^{10}$ Hence intensive dental preventative advice and careful planning and monitoring of these patients after starting medications that can cause MRONJ, must be at the forefront of care.

An OAF is a pathological communication between the oral cavity and the maxillary sinus characterised by an epithelial lining. The most likely cause is the failed primary closure of an oroantral communication secondary to a dental extraction, however could also be related to dental infection, trauma, osteomyelitis, cysts or tumour-like growths, or radiation therapy and can be more susceptible in myelosuppressed individuals. ${ }^{11}$ The communication allows bacteria from within the oral and the maxillary sinus to pass between each other, resulting in chronic infection and inflammation of the sinus lining.

Patients may complain of pain, a foul taste, escape of air between the nose and mouth and liquid escaping through the nose on drinking. To diagnose and OAF, the Valsalva method is often used where with closed nostrils the patient attempts to force air from their nose, resulting in a hiss from the mouth. Radiographic images from an OPG or CT may also show any bony defects between the two spaces.

If a communication is found at the time of surgery and less than $2 \mathrm{~mm}$ diameter, it will most likely resolve with no intervention; those under $5 \mathrm{~mm}$ diameter often respond to a haemostatic plug and suture. Anything over $5 \mathrm{~mm}$ is likely to need surgical closure. Autogenous closure is the most widely used treatment and can involve a buccal advancement flap, a buccal fad pad flap or a palatal rotational flap. All surgical approaches carry a risk of postoperative infection, and the microflora associated within the oral cavity can increase their susceptibility.

Oral surgery undertaken on the immunocompromised patient is associated with a higher risk of complications and delayed healing. It has also been found that immunocompromised patients are more likely to acquire a bilateral sinusitis (based on CT imaging) compared with an immunocompetent patient secondary to a dental infection or periodontal disease. ${ }^{12}$ Decongestants and antibiotic regimes have been successful in managing maxillary sinusitis secondary to a chronic OAF, where surgical input would normally have been attempted. ${ }^{13}$ Furthermore, a case report for repairing an OAF on an HIVpositive patient described a successful non-surgical technique. Removal of the epithelialised tissue was followed by the wearing of a splint or cover plate to create the seal. The splint was only removed to allow cleaning, and chlorhexidine $1 \%$ gel was applied each time. This approach prevented any complications and reduced the secondary infection risk; however, this relies very strongly on patient compliance. ${ }^{14}$

This case demonstrates that a multidisciplinary team approach is essential in the dental management of a myelosuppressed patient who requires dental extractions and is at a higher risk of complications. Teeth with advanced bone loss should be extracted prior to chemotherapy as they may decline rapidly during intensive immunosuppression. This should be augmented by intensive preventative care to limit the deterioration of oral health during chemotherapy. If surgical intervention is required, careful consideration should be undertaken to ensure the least invasive approach is applied and close review is in place.

\section{Learning points}

Patients with multiple comorbidities must have effective preventative care and thorough dental assessment prior to invasive treatment.

- Multidisciplinary management is essential for patients with life-limiting comorbidities or myelosuppression presenting with oral infections.

- Conditions such as aspergillosis must be considered where there is a risk of an oroantral communication.

Contributors AP, KS and NK were all involved in the perioperative treatment of the patient and all contributed to the report.

Funding The authors have not declared a specific grant for this research from any funding agency in the public, commercial or not-for-profit sectors.

Competing interests None declared.

Patient consent for publication Obtained.

Provenance and peer review Not commissioned; externally peer reviewed.

\section{REFERENCES}

1 Khan AM, Devarakonda S, Bumma N, et al. Potential of NK cells in multiple myeloma therapy. Expert Rev Hematol 2019;12:425-35.

2 Palakshappa SG, Wadhwan V, Bansal V, et al. Multiple myeloma presenting as an unhealed extraction socket: report of a case with brief review of literature. J oral Maxillofac Pathol. May-Aug 2018;22:284.

3 Gupta M, Pal R, Tikoo D. Multiple myeloma: the disease and its treatment. Int J Basic Clin Pharmacol 2013;2:103-21.

4 Davies D. Pulmonary aspergillosis. Can Med Assoc J 1963;89:392-5.

5 Warris A. The biology of pulmonary Aspergillus infections. J Infect 2014;69:S36-41.

6 Raman A, Papagiannopoulos P, Kuhar HN, et al. Histopathologic features of chronic sinusitis precipitated by odontogenic infection. am J Rhinol allergy. Mar 2019;33:113-20.

7 Kalra S, Jain V. Dental complications and management of patients on bisphosphonate therapy: a review article. J Oral Biol Craniofac Res 2013;3:25-30.

8 Peterson DE, Schimpff SC. Aspergillus sinusitis in neutropenic patients with cancer: a review. Biomed Pharmacother 1989;43:307-12.

9 Teah MJ, Syme SLE, Scheper M, et al. The care and management of bisphosphonate-associated osteonecrosis of the jaw in the patient with multiple myeloma: a case study. J Dent Hyg 2013;87:181-7.

10 McGowan K, Acton C, Ivanovski S, et al. Systemic comorbidities are associated with medication-related osteonecrosis of the jaws: case-control study. Oral Dis 2019;25:1107-15. 
11 Parvini P, Obreja K, Sader R, et al. Surgical options in oroantral fistula management: a narrative review. Int J Implant Dent 2018:4:40.

12 McCarty JL, David RM, Lensing SY, et al. Root Cause Analysis: An Examination of Odontogenic Origins of Acute Maxillary Sinusitis in Both Immunocompetent \& Immunocompromised Patients. J Comput Assist Tomogr 2017;41:484-8.
13 Mishra AK, Sinha VR, Nilakantan A, et al. Rhinosinusitis associated with post-dental extraction chronic oroantral fistula: outcomes of non-surgical management comprising antibiotics and local decongestion therapy. J Laryngol Otol 2016;130:545-53.

14 Logan RM, Coates EA. Non-Surgical management of an oro-antral fistula in a patient with HIV infection. Aust Dent J 2003;48:255-8.

Copyright 2020 BMJ Publishing Group. All rights reserved. For permission to reuse any of this content visit

https://www.bmj.com/company/products-services/rights-and-licensing/permissions/

BMJ Case Report Fellows may re-use this article for personal use and teaching without any further permission.

Become a Fellow of BMJ Case Reports today and you can:

- Submit as many cases as you like

Enjoy fast sympathetic peer review and rapid publication of accepted articles

- Access all the published articles

Re-use any of the published material for personal use and teaching without further permission

Customer Service

If you have any further queries about your subscription, please contact our customer services team on +44 (0) 2071111105 or via email at support@bmj.com.

Visit casereports.bmj.com for more articles like this and to become a Fellow 